\title{
Early outcomes of thrombolysis for acute ischaemic stroke in a South African tertiary care centre
}

\author{
Sean Wasserman, Alan Bryer
}

Background. Stroke is an important cause of death and disability in sub-Saharan Africa. Recombinant tissue plasminogen activator (tPA) thrombolysis is effective in treating acute ischaemic stroke, but may not be a viable option in developing countries.

Methods. We assessed the short-term outcomes and safety of tPA for the treatment of stroke at Groote Schuur Hospital from the year 2000. Patients with a clinical diagnosis of acute stroke with onset of stroke symptoms within 4.5 hours of receiving thrombolysis were included. Exclusion criteria were based on the National Institute of Neurological Disorders and Stroke (NINDS) rt-PA trial protocol (upper age limit was 75 years). Primary outcomes were the proportion of patients achieving significant early neurological recovery defined as an improvement of 4 or more points on the National Institutes of Health stroke scale (NIHSS) score and functional independence defined as a modified Rankin score of 2 or less at discharge. The primary safety measures were the rates of symptomatic intracranial haemorrhage (SICH) and death.

Results. From January 2000 to February 201142 patients were thrombolysed, with a mean time to tPA infusion of 160 minutes (standard deviation (SD) 50; range 60 - 270). By discharge the median NIHSS score fell from 14 (interquartile range (IQR) 10.5 17) to 7.5 (IQR 1 - 15); 28 (66.7\%) achieved significant neurological improvement, and 17 (40.5\%) were functionally independent. Two patients $(4.8 \%)$ suffered SICH and there were $3(7.1 \%)$ deaths.

Conclusion. Thrombolysis in routine clinical practice in a South African setting has similar safety and early efficacy outcomes to controlled trials and open-label studies in developing and developed countries.

S Afr Med J 2012;102(6):541-544.
Stroke is an important cause of death and disability in sub-Saharan Africa, where it was estimated that 355000 stroke deaths occurred in $2001,{ }^{1}$ with a similar age-specific prevalence to developed countries, ${ }^{2}$ but resulting in much higher levels of disability. ${ }^{3}$ Cerebrovascular disease is a growing problem in South Africa, ${ }^{4}$ and places a heavy burden on family carers in poor socioeconomic environments. ${ }^{5}$

Thrombolysis with recombinant tissue plasminogen activator (tPA, alteplase) is the only effective specific treatment for acute ischaemic stroke. The landmark National Institute of Neurological Disorders and Stroke (NINDS) rt-PA trial ${ }^{6}$ in the USA in 1995 demonstrated that patients receiving this intervention were 30\% more likely to survive with minimal disability resulting in a $12 \%$ absolute increase in the proportion having excellent functional outcomes at 3 months. The benefits of thrombolysis have been consistently reproduced when used in routine clinical practice across different patient populations. ${ }^{7-9}$ Although its use increases rates of intracranial haemorrhage, thrombolysis has no impact on overall mortality. ${ }^{10}$

Net benefit can be achieved if TPA is administered within 4.5 hours of onset of stroke symptoms. ${ }^{11}$ This narrow window of opportunity represents the largest obstacle to more widespread use because of inefficient public transport systems and low levels of awareness about stroke. ${ }^{12}$ This and other issues, such as cost and the availability of resources required to administer this treatment, have caused concerns that thrombolysis may not be a viable option for treating

Department of Medicine, Groote Schuur Hospital and University of Cape Town Sean Wasserman, $\mathrm{MB} \mathrm{ChB}$

Stroke Unit, Division of Neurology, Department of Medicine, Groote Schuur Hospital and University of Cape Town

Alan Bryer, PhD stroke in developing countries. However, there is growing evidence of of its comparable efficacy and safety when used in these settings. ${ }^{13-17}$

The new South African national stroke guidelines recommend tPA as treatment for acute ischaemic stroke within 4.5 hours of symptom onset. ${ }^{18}$ However, there are no published data on the use of thrombolysis in Africa. We aimed to evaluate the early outcomes and safety of this intervention in a South African setting.

\section{Methods}

This was a prospective, open, observational study of all patients receiving tPA for acute ischaemic stroke at Groote Schuur Hospital (GSH) from the year 2000. The hospital has a comprehensive stroke unit as defined by the national stroke guidelines.

Patient eligibility was defined by the Stroke Unit protocol and included patients with a clinical diagnosis of acute stroke in whom it was ascertained that the time of onset of stroke symptoms was within 3 hours of receiving thrombolysis. After publication of the ECASS III trial $^{19}$ in 2008 this time window was extended to 4.5 hours. Exclusion criteria were based on the NINDS rt-PA trial ${ }^{6}$ protocol (in our study the upper age limit was 75 years), which broadly restricts eligibility to those without evidence of intracranial blood on baseline computed tomography (CT) scanning, low risk of bleeding, and those without rapidly resolving symptoms or severe neurological deficit (National Institutes of Health stroke scale (NIHSS) $>25$ ).

Baseline and demographic characteristics, stroke type and severity, time intervals, risk factors, and medication history were recorded. Neurological impairment and functional disability were assessed on arrival and at discharge using the NIHSS score and the modified Rankin scale (mRS), respectively. CT scans were performed at baseline and within 36 hours of thrombolysis, or on evidence of clinical deterioration.

The primary outcome measure was the proportion of patients achieving significant early neurological recovery defined as an improvement of 4 or more points on the NIHSS score at discharge. The safety endpoint was the rate of symptomatic intracranial haemorrhage $(\mathrm{SICH})$ and death. SICH was defined according to the NINDS rt-PA/Cochrane criteria ${ }^{6,20}$ as any new evidence of 
intracranial blood on CT or magnetic resonance imaging (MRI) accompanied by a neurological deterioration of 4 or more points on the NIHSS score from baseline. Functional independence (i.e. an mRS score of 0 - 2) or an improvement of two or more points on the mRS achieved at discharge were secondary outcomes.

The proportion of patients with $\mathrm{SICH}$, mortality, independence, and neurological improvement were calculated and compared with the corresponding proportions in the alteplase arm of the NINDS rt-PA and ECASS III trials, and with observational studies in developed and other developing countries.

Demographic profiles were compared with a cohort of unmatched patients who suffered ischaemic strokes but who were managed conservatively in our stroke unit. The latter patients were ineligible for thrombolysis, and their data were recorded in the same register as those who received tPA from January 2000 to April 2006.

Written informed consent was obtained either from the patients or from a close family member. Alteplase was administered in consultation with the attending neurologist or stroke physician. An intravenous (IV) dose of alteplase $(0.9 \mathrm{mg} / \mathrm{kg}$, with $10 \%$ given as a bolus followed by an infusion over 1 hour) was administered in the emergency department after the baseline CT scan was reviewed. Selected patients received intra-arterial (IA) alteplase.

This study was approved by the UCT-GSH Human Research Ethics Committee (Ref: 386/2011).

\section{Results}

Forty-two patients were thrombolysed at GSH from January 2000 to February 2011. Their mean age was 60 (SD 12.26, range 24 - 79) and the median baseline NIHSS score was 14 (interquartile range (IQR) 10.5 - 17). Approximately $60 \%$ were clinically assessed to have largevessel atheroembolic aetiologies. Thirty-six $(85.7 \%)$ received IV tPA, $4(9.5 \%)$ received IA tPA and $2(4.8 \%)$ received bridging treatment with IV tPA followed by an endovascular procedure including IA tPA. Three (7.1\%) patients were over the age of 75 years.

Table 1. Baseline clinical characteristics of patients receiving tPA $(N=42)$

\begin{tabular}{ll}
\hline Variable & \\
\hline Age (years), median (IQR) & $62(52-67.5)$ \\
Male sex, \% & 54.8 \\
Married, $n$ (\%) & $24(57.1)$ \\
Systolic blood pressure (mmHg), median (IQR) & $149(134-170)$ \\
Diastolic blood pressure (mmHg), median (IQR) & $84(76-90)$ \\
Degree of neurological severity (NIHSS) & $14(10.5-17)$ \\
$\quad$ Mild (0 - 7), $n(\%)$ & $2(4.8)$ \\
$\quad$ Moderate (8 - 14), $n(\%)$ & $19(45.2)$ \\
$\quad$ Severe ( $\geq 15), n(\%)$ & $18(42.9)$ \\
Cause of stroke & \\
$\quad$ Large-vessel atheroembolic, $n(\%)$ & $25(59.5)$ \\
Lacunar, $n(\%)$ & $3(7.1)$ \\
Cardioembolic, $n(\%)$ & $9(21.4)$ \\
Other, $n(\%)$ & $3(7.1)$ \\
$\quad$ Unknown, $n(\%)$ & $2(4.8)$ \\
Stroke onset to treatment time (min), & $160( \pm 50)$ \\
mean (SD) &
\end{tabular}

Table 2. Demographic characteristics of patients receiving tPA compared with unmatched patients with acute ischaemic stroke managed conservatively

\begin{tabular}{lll}
\hline & $\begin{array}{l}\text { tPA } \\
(\mathbf{N = 4 2})\end{array}$ & $\begin{array}{l}\text { Non-tPA } \\
(\mathbf{N = 8 8 2})\end{array}$ \\
\hline Education & & \\
$\quad$ School (years), median (IQR) & $10(8.5-12)$ & $8(6-10)$ \\
$\quad$ Tertiary, $n$ (\%) & $6(14.3)$ & $57(6.5)$ \\
Income & & \\
$\quad$ Employed, $n(\%)$ & $17(40.5)$ & $228(25.9)$ \\
$\quad$ Grant, $n(\%)$ & $20(47.6)$ & $432(49)$ \\
Housing & & \\
$\quad$ House, $n(\%)$ & $41(97.6)$ & $736(83.4)$ \\
Shack, $n(\%)$ & $1(2.4)$ & $96(10.8)$ \\
& &
\end{tabular}

Table 1 shows clinical data, including stroke type and severity. NIHSS data were complete at discharge in 39 (93\%) patients and the $\mathrm{mRS}$ was recorded for all patients. The mean time to $\mathrm{tPA}$ infusion was 160 minutes (SD 50, range 60 - 270). Most patients (29, 72.5\%) were thrombolysed within 180 minutes. Median duration of hospital stay was 12 days (IQR 8 - 15.8). Table 2 compares demographic data of the patients receiving $\mathrm{tPA}$, and unmatched patients with acute ischaemic stroke managed conservatively in the same unit. Of the patients who received thrombolysis, 25/42 (59.5\%) arrived at hospital using their own transport; the next most common referral source was from private hospitals (5/42, 11.9\%) (Fig.1).

Fig. 2 illustrates early neurological outcomes grouped by severity.

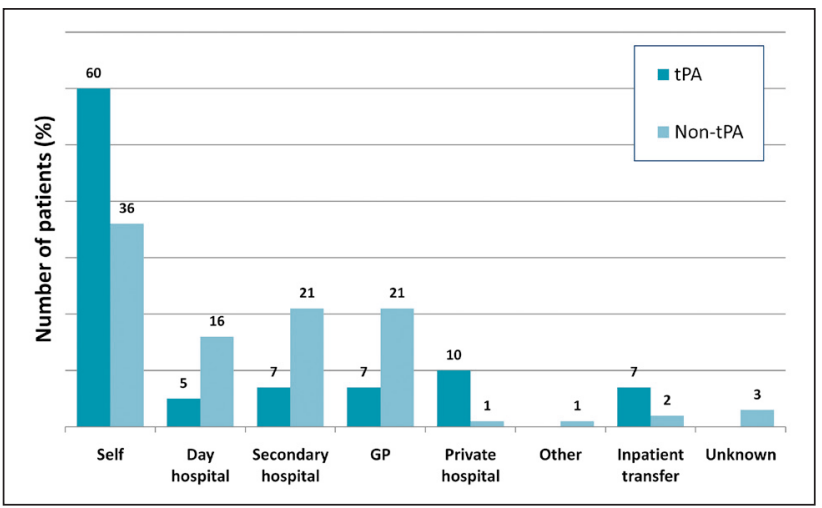

Fig. 1. Referral sources of patients receiving tissue plasminogen activator (tPA) and those managed conservatively.

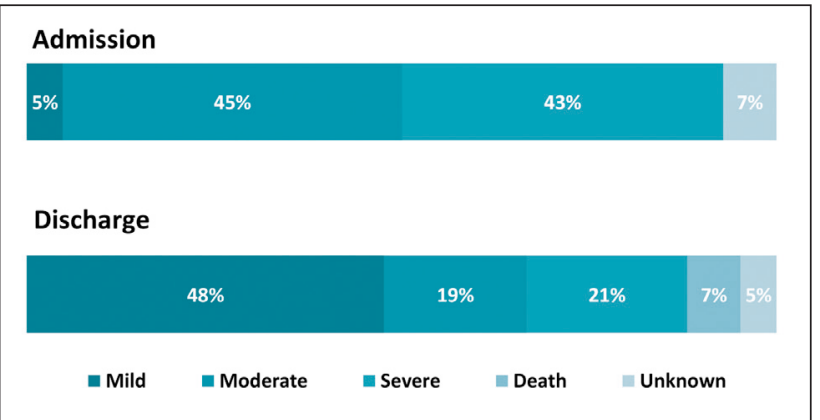

Fig. 2. Early neurological outcomes grouped by severity ( mild = NIHSS $0-7$; moderate $=$ NIHSS $8-14$; severe $=$ NIHSS $\geq 15$ ). 


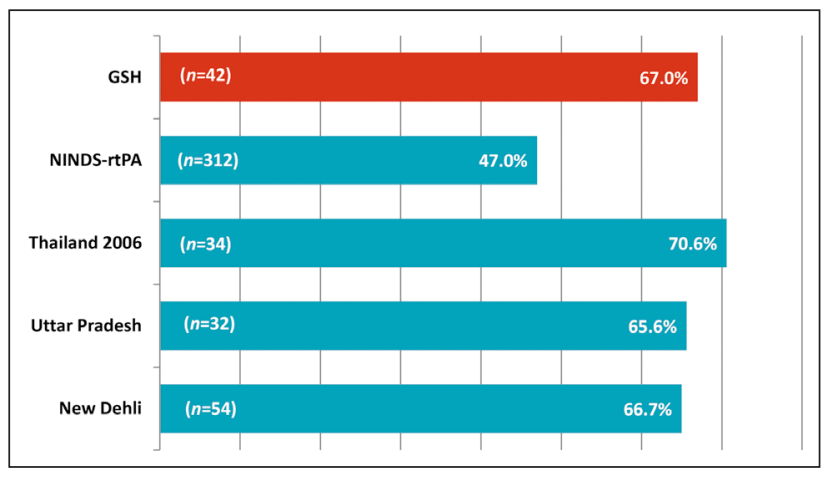

Fig. 3. Proportion of patients achieving early neurological improvement (NIHSS $\geq 4$ points) compared with other studies. ${ }^{6,13,14,16}$

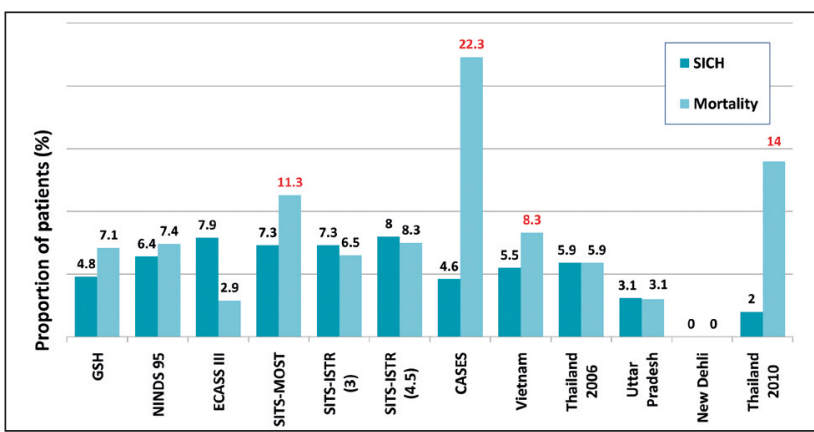

Fig. 4. Proportion of patients with symptomatic intracranial haemorrhage and mortality in the GSH cohort and other trials, ${ }^{6,19}$ as well as observational studies. ${ }^{7-9,13-17}$ SICH $=$ symptomatic intracranial haemorrhage. Percentages in red $=3-$ month data, in black = data at discharge.

The median NIHSS score fell to 7.5 (IQR 1 - 15) by the time of discharge. Forty-eight per cent of patients scored in the mild range (0 - 7), compared with only $5 \%$ on arrival. At discharge 18 (42.9\%) patients had improved their mRS by 2 or more points and 17 (40.5\%) were functionally independent. Over half (53.8\%) of our patients were discharged home. Fig. 3 compares the proportion of patients who achieved significant neurological improvement in this study with that of other studies.

Fig. 4 compares our patients and results from other studies for SICH (using the NINDS rt-PA/Cochrane definition) and mortality. Only $2(4.8 \%)$ of our patients suffered SICH. Adverse effects were not significantly related to time of tPA infusion, age or stroke severity. All three patients aged $>75$ years experienced bleeding complications with one, aged 76, suffering a symptomatic intracranial haemorrhage (NIHSS 8). Another, aged 79, had an asymptomatic intracranial haemorrhage (NIHSS 28), and the third patient, aged 78, developed a femoral haematoma (NIHSS 19).

At the time of discharge a total of $3(7.1 \%)$ patients had died, all of whom had admission Rankin scores of 5 . Besides the single patient with fatal intracranial haemorrhage the deaths were unrelated to treatment; one patient died of an acute myocardial infarction, and the other from an additional cerebral infarct and nosocomial pneumonia.

\section{Discussion}

Two-thirds of our patients achieved significant neurological improvement after receiving tPA, which compares favourably with the NINDS rt-PA trial. ${ }^{6}$ Smaller studies from other developing countries reported similar results. ${ }^{13-17}$ The European Medicines Evaluation Agency allowed registration of tPA for acute stroke in 2002, on condition that an observational safety study be performed to address concerns about the applicability of data from randomised controlled trials to individuals in daily clinical practice. At 3-month followup, $54.8 \%$ of patients in this SITS-MOST cohort were functionally independent, ${ }^{7}$ compared with $40.5 \%$ at the time of discharge in our study. Although other studies demonstrated sustained clinical benefit of thrombolysis, ${ }^{20}$ this could not be assessed with our study design which was limited by a short follow-up period. However, the functional outcomes of our patients may be comparable with those of the SITS-MOST study as improvement after tPA typically occurs early because of vessel recanalisation, and further recovery would be anticipated in our surviving patients with rehabilitation in the 90 days post discharge.

Our patients had a lower rate of SICH compared with the NINDS rt-PA trial $(4.8 \% \text { v. } 6.4 \%)^{6}$ and the large open-label SITS-ISTR study (8.0\% for patients treated between 3 and 4.5 hours and $7.3 \%$ for patients treated within 3 hours). ${ }^{9}$ Pooled data from five observational studies from developing countries show a lower proportion of patients suffering SICH, 9/293 (3.1\%), but the rates ranged from 0 to $5.9 \%$ between the different centres. ${ }^{13-17}$ Although the number is small and does not represent a significant association, all three of our patients over the age of 75 had bleeding complications. Studies in the developed world support the safety of tPA in patients over 80 years. ${ }^{21}$ However, more caution may be needed in our setting when thrombolysing elderly patients.

Our in-patient mortality was similar to that of the NINDS rt-PA trial (7.4\%), but could not be compared with large observational studies such as SITS-MOST and CASES as these only reported 3-month mortality rates. The 7-day mortality of 11621 patients treated within 3 hours in routine practice across Europe was similar to ours at $6.5 \%$; the rate for those treated between 3 and 4.5 hours in the same registry was 7.5\%. ${ }^{9}$ In 3 studies from developing countries the mortality at discharge ranged from 0 to $5.9 \% \cdot{ }^{13,14,16}$

It is estimated that $<2 \%$ of patients with ischaemic stroke in our hospital drainage area received thrombolysis, which seems comparable with data from other developing countries. ${ }^{15,16}$ However, our reported thrombolysis rate is an overestimation for the general population, as most patients with acute stroke are managed conservatively at lower level facilities where thrombolysis is not available.

This study was not designed to identify barriers to accessing thrombolysis, but inferences may be drawn from our findings in this regard. Most patients who qualified for tPA used their own transport to hospital, suggesting that delays in the emergency medical services transport system may be a cause for arrival outside of the 4.5 hour window period. A greater proportion of patients who received thrombolysis were employed, and lived in brick houses compared with conservatively managed patients, suggesting that those with higher incomes are more likely to arrive at hospital within the narrow time window required for this intervention. As in other studies, ${ }^{10,12}$ low levels of public awareness of stroke may be another barrier to thrombolysis in our setting as reflected by the difference in education levels between the patient groups. Certain quarters in South Africa's emergency medicine community ${ }^{22}$ and elsewhere are reluctant to use tPA for stroke, ${ }^{23}$ and this may also have contributed to the small numbers of patients referred from secondary level hospitals.

A study limitation is the relatively small number of patients with ischaemic stroke in the GSH cohort that received tPA. Nevertheless, the data indicate that the use of thrombolysis in routine clinical practice in a South African setting has similar safety and early efficacy outcomes to developed and other developing countries Thrombolysis is specialised and is not available to most South Africans. However, its benefits for reducing disability for stroke 
victims should be promoted in the lay and medical community. Implementing an acute stroke response network in the emergency services must be considered to facilitate early arrival and assessment at a designated stroke centre where thrombolysis is available.

\section{References}

1. Connor MD, Walker R, Modi G, Warlow CP. Burden of stroke in black populations in sub-Saharan Africa. Lancet Neurol 2007:6:269-278.

2. Connor MD, Thorogood M, Casserly B, Dobson C, Warlow CP, SASPI Project Team. Prevalence of stroke survivors in rural South Africa: results from the Southern Africa Stroke Prevention Initiative (SASPI) Agincourt field site. Stroke 2004;35:627-632.

3. Bonita R, Solomon N, Broad JB. Prevalence of stroke and stroke-related disability. Estimates from the Auckland stroke studies. Stroke 1997:28:1898-1902.

4. Tollman SM, Kahn K, Sartorius B, Collinson MA, Clark S), Garenne ML. Implications of mortality transition for primary health care in rural South Africa: a population-based surveillance study. Lancet 2008;372:893-901

5. Wasserman S, de Villiers L, Bryer A. Community-based care of stroke patients in a rural African setting. S Afr Med J 2009;99:579-583.

6. The National Institute of Neurological Disorders and Stroke rt-PA Stroke Study Group. Tissue plasminogen activator for acute ischemic stroke. N Engl J Med 1995;333:1581-1587.

7. Wahlgren N, Ahmed N, Davalos A, et al. Thrombolysis with alteplase for acute ischaemic stroke in the Safe Implementation of Thrombolysis in Stroke-Monitoring Study (SITS-MOST): an observational study Lancet 2007:369:275-282.

8. Hill MD, Buchan AM, Canadian Alteplase for Stroke Effectiveness Study (CASES) Investigators. Thrombolysis for acute ischemic stroke: results of the Canadian Alteplase for Stroke Effectiveness Study. Can Med Assoc J 2005;172:1307-1312.

Study. Can Med Assoc J 2005;172:1307-1312.
9. Wahlgren N, Ahmed N, Dávalos A, et al. Thrombolysis with alteplase $3-4.5 \mathrm{~h}$ after acute ischaemic Wahlgren N, Ahmed N, Dávalos A, et al. Thrombolysis with alteplase 3 -
stroke (SITS-ISTR): an observational study. Lancet 2008:372:1303-1309.

10. Bambauer KZ, Johnston SC, Bambauer DE, Zivin JA. Reasons why few patients with acute stroke receive tissue plasminogen activator. Arch Neurol 2006:63:661-664.

11. Lees KR, Bluhmki E, von Kummer R, et al. Time to treatment with intravenous alteplase and outcome in stroke: an updated pooled analysis of ECASS, ATLANTIS, NINDS, and EPITHET trials. Lancet 2010;375:1695-1703.

12. Kwan J, Hand P, Sandercock P. A systematic review of barriers to delivery of thrombolysis for acute stroke. Age Ageing 2004;33:116-121.

13. Sharma SR, Sharma N. Hyperacute thrombolysis with recombinant tissue plasminogen activator of acute ischemic stroke: feasibility and effectivity from an Indian perspective. Ann Indian Acad Neurol 2008;11: 221-224.

14. Padma MV, Singh MB, Bhatia R, et al. Hyperacute thrombolysis with IV rtPA of acute ischemic stroke: efficacy and safety profile of 54 patients at a tertiary referral center in a developing country. Neurol

15. Nguyen TH, Truong AL, Ngo MB, et al. Patients with thrombolysed stroke in Vietnam have an excellent outcome: results from the Vietnam Thrombolysis Registry. Eur J Neurol 2010;17:11881192

16. Suwanwela NC, Phanthumchinda K, Likitjaroen Y. Thrombolytic therapy in acute ischemic stroke in Asia: The first prospective evaluation. Clin Neurol Neurosurg 2006;108:549-552.

7. Muengtaweeponsa, S, Dharmasaroja S, Kummark U. Outcomes of intravenous thrombolytic therapy for acute of a community-based hospital in a developing country. Journal of Stroke and Cerebrovascular Diseases 2012 (in press)

18. Bryer A, Connor M, Haug P, et al. South African guideline for management of ischaemic stroke and transient ischaemic attack 2010: a guideline from the South African Stroke Society (SASS) and the SASS Writing Committee. S Afr Med J 2010;100 Pt 2:747-778.

19. Hacke W, Kaste M, Bluhmki E, et al. Thrombolysis with alteplase 3 to 4.5 hours after acute ischemic stroke. N Engl J Med 2008;359:1317-1329.

20. Wardlaw JM Murray V, Berge E del Zoppo GJ. Thrombolysis for acute ischaemic stroke. Cochrane Database of Systematic Reviews 2009, Issue 4.

21. Berrouschot J, Rother J, Glahn J, Kucinski T, Fiehler I, Thomalla G. Outcome and severe hemorrhagic complications of intravenous thrombolysis with tissue plasminogen activator in very old $(>$ or $=80$ years) stroke patients. Stroke 2005:36:2421-2425.

22. Lahri S, Wallis L. South African ischaemic stroke guideline, 2010 (Letter). S Afr Med J 2011;101:7.

23. Brown DL, Barsan WG, Lisabeth LD, Gallery ME, Morgenstern LB. Survey of emergency physician about recombinant tissue plasminogen activator for acute ischemic stroke. Ann Emerg Me 2005; $46: 56-60$

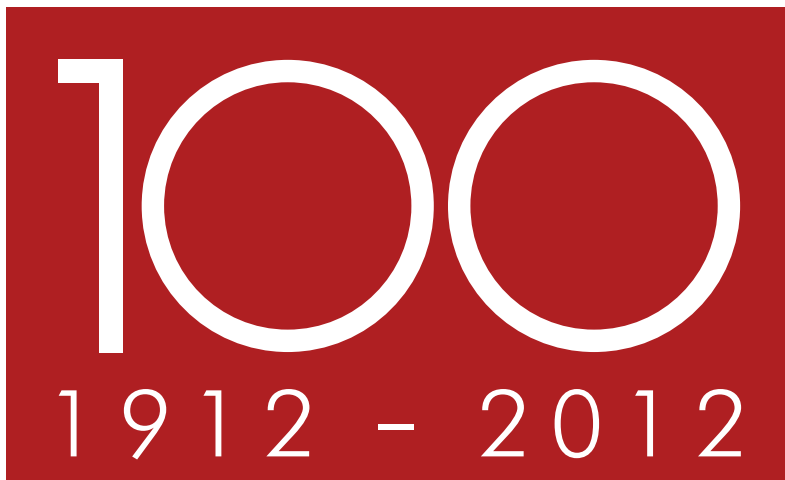

\section{BUILDING THE FUTURE}

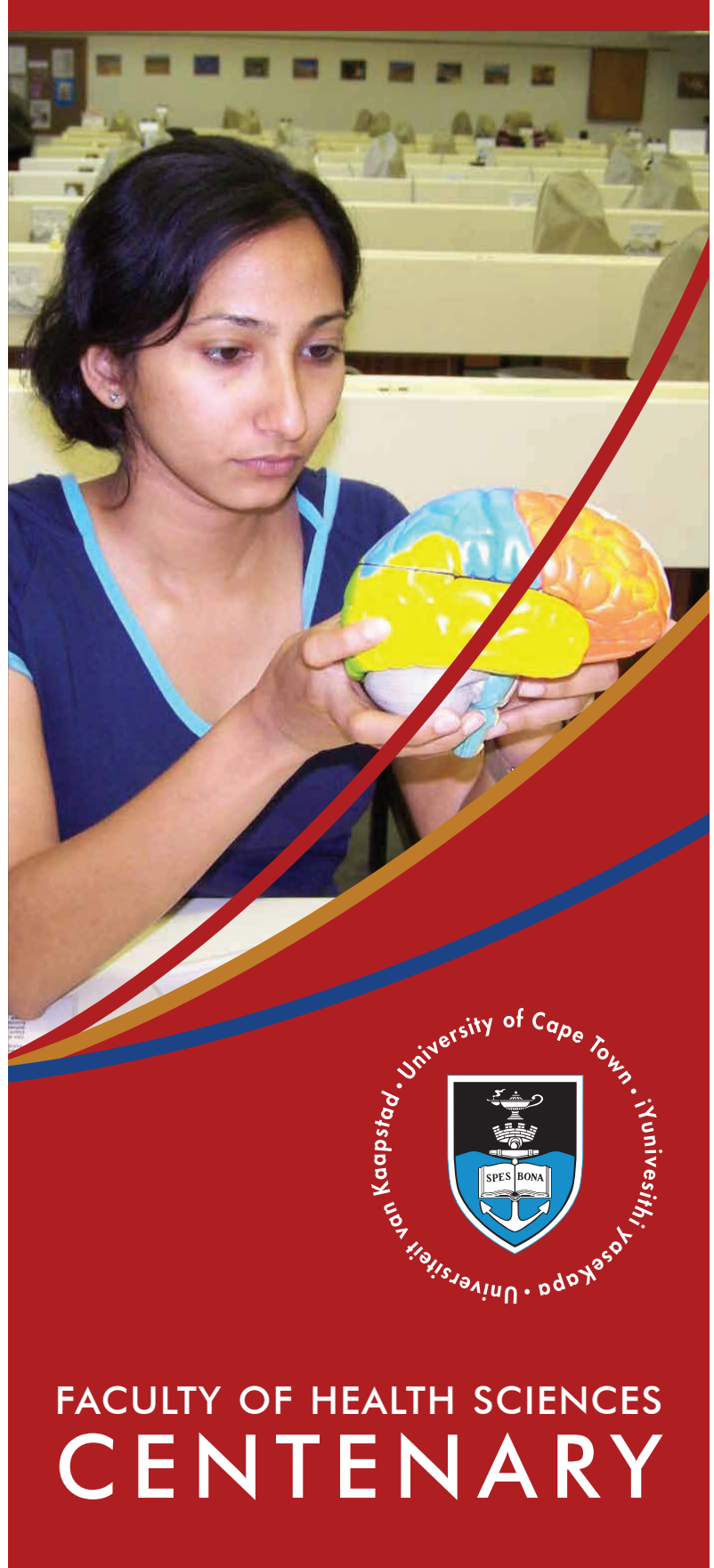

